\title{
Association between Healthcare Utilization and Depression in Korean Women with Cardiovascular Conditions
}

\author{
Se Jin Park', Jung Won Jang ${ }^{1,2}$, Ah Young Kim¹, Seungyeon Hong', \\ Boram Yuk ${ }^{1}$, Ye Won Min ${ }^{1}$, Kyung A Park ${ }^{1,2}$, and Subin Park ${ }^{1}$ \\ ${ }^{1}$ Department of Research Planning, Mental Health Research Institute, National Center for Mental Health, Seoul, Republic of Korea \\ ${ }^{2}$ Department of Health Sciences, Graduate School, Hanyang University, Seoul, Republic of Korea
}

Objective This study aimed to examine the associations between depression and both coronary artery disease (CAD) and cardiovascular risk factors (CVRs) in Korean women. Furthermore, this study sought to determine whether depression was associated with use of healthcare services in women with CAD or CVRs.

Methods This cross-sectional study was conducted on 26,335 women who were aged 19 years or older, and who participated in the Korean National Health and Nutrition Examination Survey (2007-2014). Associations of prior diagnosis of depression with CAD and CVRs and with nonutilization of healthcare services were investigated.

Results Women with depression had a higher prevalence of CAD and CVRs including obesity, hypertension, dyslipidemia, and metabolic syndrome than those without depression. In addition, depression was significantly associated with nonutilization of healthcare services in women with most CVRs.

Conclusion Considering the high rate of comorbid depression with CAD or CVRs and the low levels of health service utilization in depressed patients, screening for common CVRs, such as obesity, hypertension, and dyslipidemia, should be provided for patients with depression in mental health care settings.

Psychiatry Investig 2017;14(6):801-807

Key Words Coronary artery disease, Cardiovascular risk factors, Depression, Healthcare utilization.

\section{INTRODUCTION}

Depression and cardiovascular disease are currently the 2 most common causes of disability in high-income countries and expected to become so for countries of all income levels by 2030. ${ }^{1}$ Previous studies have shown that depression is associated with increased risk of cardiovascular disease ${ }^{2}$ and an independent major risk factor for the onset of a wide range of cardiovascular diseases and cardiac mortality.,4 In addition, many studies have reported causative relationships between depression and hypertension, ${ }^{5}$ diabetes, ${ }^{6}$ obesity ${ }^{7,8}$ dyslipidemia, ${ }^{8}$

Received: November 1, 2016 Revised: January 28, 2017

Accepted: March 2, 2017 Available online: September 29, 2017

$\triangle$ Correspondence: Subin Park, MD, PhD

Department of Research Planning, Mental Health Research Institute, National Center for Mental Health, 127 Yongmasan-ro, Gwangin-gu, Seoul 04933, Republic of Korea

Tel: +82-2-2204-0108, Fax: +82-2-2204-0393, E-mail: subin-21@hanmail.net

(a) This is an Open Access article distributed under the terms of the Creative Commons Attribution Non-Commercial License (http://creativecommons.org/licenses/by$\mathrm{nc} / 4.0$ ) which permits unrestricted non-commercial use, distribution, and reproduction in any medium, provided the original work is properly cited. and metabolic syndrome, ${ }^{9,10}$ which are known to be cardiovascular disease risk factors (CVRs). Moreover, several studies have reported a clear sex difference in the relationship between depression and cardiovascular disease, ${ }^{4}$ for example, in one study, ${ }^{4}$ women with depression were at significantly greater risk of cardiac mortality relative to men with depression.

Depression is a risk factor not only for cardiovascular disease but also for worse prognoses. Comorbid depression and cardiovascular disease increase medical costs ${ }^{11}$ and healthcare utilization. ${ }^{12}$ Comorbid depression and coronary artery disease $(\mathrm{CAD})$ are associated with higher hospital readmission rates and increased overall and outpatient healthcare costs. ${ }^{13}$ Depression in congestive heart failure patients is also an independent predictor of mortality and rehospitalization..$^{14} \mathrm{In}$ addition, nonadherence to medication regimens is associated with significant clinical and economic burden ${ }^{15}$ and the rate of medication nonadherence in patients with comorbid depression and coronary heart disease is double that of nondepressed patients with coronary heart disease. ${ }^{16}$

Given the high comorbidity rate for depression and cardio- 
vascular disease or CVRs, and the resultant disease burden, it is important to understand the impact of depression on healthcare utilization in patients with comorbid depression and cardiovascular disease or CVRs. Therefore, this study aimed to examine the associations between depression and CAD and CVRs in women aged 19 years or older, based on nationally representative data collected in Korea. Furthermore, the study sought to determine whether depression was associated with increased or decreased use of healthcare services in women with CAD or CVRs.

\section{METHODS}

\section{Data source and study sample}

This study used cross-sectional data from the Korean National Health and Nutrition Examination Survey (KNHANES) conducted by the Korea Centers for Disease Control and Prevention between 2007 and 2014. The KNHANES targeted the noninstitutionalized Korean civilian population and monitored trends in the prevalence, awareness, treatment, and control of selected chronic diseases; assessed trends in nutritional status and risky behavior; and analyzed risk factors for chronic diseases. The survey used a stratified multistage probability sampling strategy based on geographical region, sex, and age, and consisted of a health interview survey, health examination survey, and nutrition survey. The health examination survey was conducted in local community health centers and clinics, and standardized protocols were used to assess anthropometry, blood pressure, and laboratory indices. The Institutional Review Board at the Korea Centers for Disease Control and Prevention approved the survey protocol (2007-02CON-04-P, 200804EXP-01-C, 2009-01CON-03-2C, 2010-02CON-21-C, 201102CON-06-C, 2012-01EXP-01-2C, 2013-07CON-03-4C, and 2013-12EXP-03-5C). All participants of the KNHANES used in the current study provided written informed consent. The KNHANES data used in the present study is freely available. ${ }^{17}$

In total, 50,094 adults aged 19 years or older were included in the KNHANES IV (2007-2009), V (2010-2012), and VI (20132014); of these, 45,598 participated in the health interview and examination surveys, with a participation rate of $91.02 \%$. We excluded 19,263 men from a total of 45,498 participants, and ultimately, 26,335 women aged 19 years or older were included in the present study.

\section{Measures}

\section{Definition of depression}

Women who reported having been diagnosed with depression by a physician at some point during their lives were considered depressed; therefore, for the purposes of this study, the definition of depression included both previous and current depression. The survey subjects were asked the following questions: "Have you ever had depression?" followed by "Have you ever been diagnosed with depression by a physician?" The cases involving depression were limited to those in which women answered "yes" to both questions. ${ }^{17}$

\section{Definition of CAD}

CAD was considered present if subjects reported angina or myocardial infarction, which were assessed in the health interview, using a dichotomous (responses of "yes" or "no") question: "Have you ever been diagnosed with angina or myocardial infarction by a physician?"

\section{Definition of CVRs}

CVRs included obesity, hypertension, diabetes, dyslipidemia, and metabolic syndrome, which were measured during the health examination. Anthropometric measurements included height, body weight, body mass index (BMI), and waist circumference. Height was measured to the nearest 0.1 $\mathrm{cm}$ with the subject standing barefoot. Body weight was measured to the nearest $0.1 \mathrm{~kg}$ using a balance scale. Waist circumference was measured to the nearest $0.1 \mathrm{~cm}$ at the midpoint between the subcostal and suprailiac landmarks, using a fiberglass tape. Health professionals measured blood pressure 3 times, using a mercury sphygmomanometer, after subjects had rested for 5 minutes in a stable state. The mean values for the second and third measurements of systolic blood pressure (SBP) and diastolic blood pressure (DBP) were included in the analysis. Blood samples were collected from the antecubital vein after subjects had fasted for at least 8 hours. Fasting plasma glucose (FPG), total cholesterol (TC), triglycerides (TG), high-density lipoprotein cholesterol (HDL-C), and low-density lipoprotein cholesterol (LDL-C) were measured using an automated hematology analyzer [ADVIA 1650 (Siemens, USA) in 2007; Hitachi automatic analyzer 7600 (Hitachi, Japan) during 2008-2010] at a laboratory at the Seoul Medical Science Institute (Seoul, Korea) in 2007 and at the Neodin Medical Institute (Seoul, Korea) during 2008-2012.

BMI was calculated using measurements of subjects' weight and height [weight $(\mathrm{kg}) /$ height $\left(\mathrm{m}^{2}\right)$ ]. Obesity was considered present if subjects had a BMI of $\geq 25 \mathrm{~kg} / \mathrm{m}^{2}$. Hypertension was considered present if subjects had SBP of $\geq$ $140 \mathrm{~mm} \mathrm{Hg}$, DBP of $\geq 90 \mathrm{~mm} \mathrm{Hg}$, or a prescription for hypertension medication. Diabetes was considered present if subjects had an FPG level of $\geq 126 \mathrm{mg} / \mathrm{dL}$, diabetes diagnosed by a physician, or a prescription for diabetes medication or insulin injections. Dyslipidemia was considered present if subjects had at least 1 of the following: high total cholesterol 
( $\geq 240 \mathrm{mg} / \mathrm{dL}$ or medication for cholesterol), low HDL-C ( $<40 \mathrm{mg} / \mathrm{dL})$, high LDL-C $(\geq 160 \mathrm{mg} / \mathrm{dL}$ or medication for cholesterol), or high TG ( $\geq 200 \mathrm{mg} / \mathrm{dL}$ ) levels. Metabolic syndrome was considered present if at least 3 of the following 5 criteria were satisfied: 1 ) abdominal obesity (waist circumference of $\geq 85 \mathrm{~cm}$ ), measured using a Korean-specific standard; 2) high blood pressure (SBP of $\geq 130 \mathrm{~mm} \mathrm{Hg}$ or DBP of $\geq 85$ $\mathrm{mm} \mathrm{Hg}) ; 3)$ a high TG level ( $\geq 150 \mathrm{mg} / \mathrm{dL}) ; 4)$ a low HDL-C level ( $<50 \mathrm{mg} / \mathrm{dL})$; and 5) elevated FPG $(\geq 100 \mathrm{mg} / \mathrm{dL}) .{ }^{17} \mathrm{We}$ used the version of the National Cholesterol Education Program's Adult Treatment Panel III criteria recommended by the American Heart Association and the National Heart, Lung, and Blood Institute. ${ }^{18}$

\section{Nonutilization of healthcare services}

Participants were asked about nonutilization of healthcare services despite a requirement for medical care during the preceding year, using the question, "During the past year, have you decided not to visit a hospital or clinic center (excluding dental clinics/hospitals) despite needing medical care?" A response of "yes" indicated nonutilization of healthcare services. Healthcare services included treatment, preventive medical care (immunization, screening, and health examination), physical therapy, and rehabilitation and excluded dental health services.

\section{Covariates}

Sociodemographic characteristics included age, educational level (<high school or $\geq$ high school) and monthly household income (quartile 1, quartile 2, quartiles 3, and 4). Chronic physical conditions, assessed via the health interview, included chronic obstructive pulmonary disease, asthma, stroke, arthritis, tuberculosis, chronic renal failure, atopic dermatitis, thyroid gland, cancer, and cirrhosis of the liver. The overall number of chronic physical conditions included these 10 conditions, $1 \mathrm{CAD}$, and the 5 CVRs mentioned above and therefore ranged from 0 to 16 .

\section{Statistical analysis}

Considering that the KNHANES involved a complex sam- pling design, weighted values were applied using the surveyrelated procedure in SAS software version 9.4 (SAS Institute Inc., Cary, NC, USA) for all analyses, to produce nationally representative estimates and correct variance estimates. The prevalence rates for CAD and CVRs were age-standardized into 10-year age groups, using the standard population in the Population Projections for Korea: 2010-2016 (based on the 2010 census), and standard errors (SEs) were estimated for the age-standardized rates. Log-binomial regression was performed using PROC GENMOD procedure in SAS statistical software, to estimate adjusted prevalence ratios (APRs) for CAD and CVRs for women with and without depression after adjusting for age. To compare the general characteristics of women with CAD or CVRs according to the presence of depression, Student's t test and $\chi^{2}$ tests were performed for continuous and categorical variables, respectively. To examine the effect of depression on healthcare utilization, multivariable logistic regression was used to calculate adjusted odds ratios (AORs) and $95 \%$ confidence intervals (CIs), adjusted for age, educational level, monthly household income, and number of chronic physical conditions (0-16). The analysis model included nonutilization of healthcare services as a dependent variable and depression as an independent variable. The significance level was set at $\mathrm{p}<0.05$ for all analysis.

\section{RESULTS}

\section{Association between depression and CAD/CVRs}

Of the 26,335 women aged 19 years or older included in the study, 1,505 (5.43\%) had been diagnosed with depression by a physician. With respect to comorbidity, 994 (66.05\%) of the 1,505 women with depression and 13,746 (55.4\%) of the 24,830 women without depression had comorbid CAD or CVRs (Figure 1).

The CAD prevalence rate in women with depression $(2.1 \%$, $\mathrm{SE}=0.29)$ was approximately 1.5 times higher relative to that of those without depression $(1.46 \%, \mathrm{SE}=0.08)$ after adjusting for age (adjusted prevalence ratio $=1.471,95 \% \mathrm{CI}=1.455-1.488$ ). In addition, prevalence rates for obesity, hypertension, dyslipidemia, and metabolic syndrome in women with depression

Figure 1. Prevalence of depression and CVCs. CVCs is defined as any CAD (angina or myocardial infarction) or CVRs (obesity, hypertension, diabetes, dyslipidemia, or metabolic syndrome). n: number of study samples, \%: weighted proportion, CVCs: cardiovascular conditions, CAD: coronary artery disease, CVRs: cardiovascular disease risk factors.

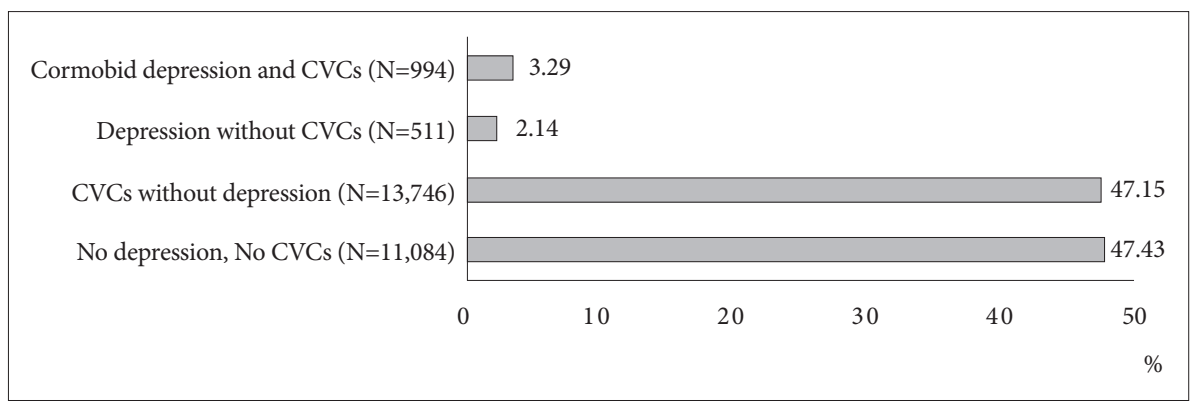


were higher relative to those observed in women without depression. However, there was no significant difference in diabetes prevalence rates between women with and without depression (Table 1).

\section{Association between depression and healthcare utilization in women with CAD or CVRs}

Women with depression were significantly older $(p<0.001)$ and reported lower educational levels $(\mathrm{p}=0.004)$ and household income ( $\mathrm{p}=0.009)$ and higher numbers of chronic physical conditions $(\mathrm{p}=0.017)$ relative to women without depression (Table 2).

Women with CAD or CVRs and comorbid depression were generally less likely to have used healthcare services despite a need for medical care during the preceding year relative to those without depression. In particular, after adjusting for

Table 1. Age-standardized prevalence (SE) and prevalence ratio $(95 \% \mathrm{Cl})$ of $\mathrm{CAD}^{*}$ and $\mathrm{CVRs}^{\dagger}$ by depression among Korean women aged 19 and over

\begin{tabular}{|c|c|c|c|c|c|c|}
\hline & \multicolumn{2}{|c|}{ With depression } & \multicolumn{2}{|c|}{ Without depression } & \multirow{2}{*}{$\operatorname{PR}(95 \% \mathrm{CI})^{\ddagger}$} & \multirow{2}{*}{$\operatorname{APR}(95 \% \mathrm{CI})^{\ddagger}$} \\
\hline & $\mathrm{N}$ & Prevalence (SE) & $\mathrm{N}$ & Prevalence (SE) & & \\
\hline$\overline{\mathrm{CAD}}$ & 65 & $2.10(0.29)$ & 511 & $1.46(0.08)$ & $1.876(1.854-1.898)$ & $1.471(1.455-1.488)$ \\
\hline Obesity & 510 & $29.89(1.68)$ & 7126 & $26.59(0.36)$ & $1.189(1.185-1.192)$ & $1.055(1.052-1.058)$ \\
\hline Hypertension & 517 & $21.71(1.03)$ & 6596 & $20.45(0.26)$ & $1.320(1.316-1.324)$ & $1.033(1.030-1.035)$ \\
\hline Diabetes & 167 & $7.74(0.80)$ & 1984 & $7.27(0.19)$ & $1.266(1.258-1.274)$ & $0.994(0.988-1.000)$ \\
\hline Dyslipidemia & 561 & $39.36(1.97)$ & 7176 & $36.16(0.44)$ & $1.198(1.195-1.201)$ & $1.036(1.034-1.038)$ \\
\hline Metabolic syndrome & 447 & $21.79(1.27)$ & 5485 & $17.85(0.28)$ & $1.393(1.388-1.398)$ & $1.099(1.095-1.102)$ \\
\hline
\end{tabular}

${ }^{*} \mathrm{CAD}$ was defined as angina or myocardial infarction, ${ }^{\dagger} \mathrm{CVRs}$ : obesity, hypertension, diabetes, dyslipidemia, metabolic syndrome, ${ }^{\ddagger} \mathrm{PR}(95 \%$ CI), APR (95\% CI) of depression vs. non-depression. SE: standard error, CI: confidence interval, CAD: coronary artery disease, CVRs: cardiovascular disease risk factors, PR: prevalence ratio, APR: age adjusted prevalence ratio

Table 2. Demographic characteristics of the samples according to the presence of depression among Korean women with CAD or CVRs

\begin{tabular}{|c|c|c|c|c|}
\hline Characteristics & $\begin{array}{c}\text { Total } \\
(\mathrm{N}=14,740)\end{array}$ & $\begin{array}{l}\text { With depression } \\
\qquad(\mathrm{N}=994)\end{array}$ & $\begin{array}{l}\text { Without depression } \\
\qquad(\mathrm{N}=13,746)\end{array}$ & p-value* \\
\hline Age, year, mean (SE) & $54.11(0.20)$ & $55.51(0.63)$ & $54.02(0.20)$ & $<0.0001$ \\
\hline Age groups, year, $\mathrm{N}(\%)^{\dagger}$ & & & & 0.0098 \\
\hline $19-29$ & $683(7.56)$ & $28(4.59)$ & $655(95.41)$ & \\
\hline $30-39$ & $1488(11.96)$ & $76(5.77)$ & $1412(94.23)$ & \\
\hline $40-49$ & $2163(18.83)$ & $115(5.55)$ & $2048(94.45)$ & \\
\hline $50-59$ & $3281(23.69)$ & $242(7.39)$ & $3039(92.61)$ & \\
\hline $60-69$ & $3555(18.68)$ & $297(7.85)$ & $3258(92.15)$ & \\
\hline$\geq 70$ & $3570(19.27)$ & $236(6.33)$ & $3334(93.67)$ & \\
\hline Education, $\mathrm{N}(\%)^{\dagger}$ & & & & 0.0043 \\
\hline$\geq$ High school & $5746(46.03)$ & $319(5.69)$ & $5427(94.31)$ & \\
\hline$<$ High school & $8929(53.97)$ & $670(7.23)$ & $8259(92.77)$ & \\
\hline Monthly household income ${ }^{\ddagger}, \mathrm{N}(\%)^{\dagger}$ & & & & 0.0095 \\
\hline$<\mathrm{Q} 1$ & $4722(27.22)$ & $368(7.73)$ & $4354(92.27)$ & \\
\hline Q1 to $<$ Q2 & $2832(19.99)$ & $201(6.78)$ & $2631(93.22)$ & \\
\hline Q2 to $<$ Q3 & $3200(24.67)$ & $206(6.39)$ & $2994(93.61)$ & \\
\hline$\geq \mathrm{Q} 3$ & $3742(28.12)$ & $207(5.41)$ & $3535(94.59)$ & \\
\hline No. of chronic physicalconditions ${ }^{\S}, \mathrm{N}(\%)^{\dagger}$ & & & & 0.0167 \\
\hline $1-2$ & $9716(68.68)$ & $594(64.19)$ & $9122(68.99)$ & \\
\hline $3-4$ & $4432(27.94)$ & $347(32.02)$ & $4085(27.66)$ & \\
\hline$\geq 5$ & $592(3.38)$ & $53(3.79)$ & $539(3.35)$ & \\
\hline
\end{tabular}

*statistical significance for difference between groups with and without depression, ${ }^{\dagger} \%$ refers to population weighted proportions, ${ }^{\ddagger}$ groups by quartiles, \$chronic physical conditions were included chronic obstructive pulmonary disease, asthma, stroke, arthritis, tuberculosis, chronic renal failure, atopic dermatitis, thyroid gland, cancer, liver cirrhosis, CAD and CVRs. SE: standard error, CAD: coronary artery disease, CVRs: cardiovascular disease risk factors 
Table 3. AOR of non-utilization of any healthcare among Korean women aged 19 and over

\begin{tabular}{|c|c|c|c|}
\hline & \multicolumn{2}{|c|}{ Non-utilization of healthcare } & \multirow{2}{*}{$\operatorname{AOR}(95 \% \mathrm{CI})^{\ddagger}$} \\
\hline & $\mathrm{N}(\%)^{*}$ & p-value ${ }^{\dagger}$ & \\
\hline $\mathrm{CAD}^{\S}$ with depression $(\mathrm{N}=65)$ & $24(38.56)$ & 0.0617 & $1.72(0.92-3.19)$ \\
\hline CAD without depression $(\mathrm{N}=511)$ & $134(26.18)$ & & Ref \\
\hline Obesity with depression $(\mathrm{N}=510)$ & $160(30.79)$ & 0.0011 & $1.48(1.15-1.92)$ \\
\hline Obesity without depression $(\mathrm{N}=7,126)$ & $1681(22.56)$ & & Ref \\
\hline Hypertension with depression $(\mathrm{N}=517)$ & $153(29.90)$ & 0.0003 & $1.50(1.18-1.91)$ \\
\hline Hypertension without depression $(\mathrm{N}=6,596)$ & $1518(21.70)$ & & Ref \\
\hline Diabetes with depression $(\mathrm{N}=167)$ & $45(26.14)$ & 0.0785 & $1.44(0.96-2.18)$ \\
\hline Diabetes without depression $(\mathrm{N}=1,984)$ & $412(19.74)$ & & Ref \\
\hline Dyslipidemia with depression $(\mathrm{N}=561)$ & $161(27.63)$ & 0.0139 & $1.30(1.02-1.67)$ \\
\hline Dyslipidemia without depression $(\mathrm{N}=7,176)$ & $1641(21.97)$ & & Ref \\
\hline Metabolic syndrome with depression $(\mathrm{N}=447)$ & $140(29.62)$ & 0.0053 & $1.42(1.09-1.84)$ \\
\hline Metabolic syndrome without depression $(\mathrm{N}=5,485)$ & $1291(22.72)$ & & Ref \\
\hline CVCs" with depression $(\mathrm{N}=994)$ & $292(28.59)$ & 0.0002 & $1.35(1.13-1.62)$ \\
\hline CVCs without depression $(\mathrm{N}=13,746)$ & $3183(22.30)$ & & Ref \\
\hline
\end{tabular}

*\% refers to population weighted proportions, ${ }^{\dagger}$ statistical significance for difference between CAD/CVRs groups with and without depression, ‡adjustment for age, education, income, and number of chronic physical conditions (0-16), \#if AORs $>1$ then less utilization of any healthcare, and AORs $<1$ then more utilization of any healthcare, ${ }^{\S} \mathrm{CAD}$ was defined as angina or myocardial infarction, "CVCs is defined as any CAD or CVRs (obesity, hypertension, diabetes, dyslipidemia, or metabolic syndrome). AOR: adjusted odds ratio, CAD: coronary artery disease, CVRs: cardiovascular disease risk factors, CI: confidence interval, CVCs: cardiovascular conditions

age, education, income, and number of chronic physical conditions, depression was positively associated with nonutilization of healthcare services in women with CVRs including obesity (AOR=1.48, 95\% CI=1.15-1.92), hypertension (AOR= $1.50,95 \% \mathrm{CI}=1.18-1.91)$, dyslipidemia $(\mathrm{AOR}=1.30,95 \% \mathrm{CI}=$ $1.02-1.67)$, and metabolic syndrome $(\mathrm{AOR}=1.42,95 \% \mathrm{CI}=$ 1.09-1.84); the result regarding diabetes was nonsignificant (Table 3).

\section{DISCUSSION}

The age-standardized prevalence rates for CAD and CVRs (with the exception of diabetes) in women with depression were significantly higher relative to those observed in women without depression. In addition, depression was significantly associated with nonutilization of healthcare services in women with most CVRs (obesity, hypertension, dyslipidemia, and metabolic syndrome).

Consistent with previous studies, depression was associated with increased $\mathrm{CAD}^{2-4}$ and risk factors for CAD development. ${ }^{5,7-10}$ A meta-analysis of 19 prospective cohort studies ( $\mathrm{n}=$ 323,709 ) reported that depression was associated with a significant increase in risk of myocardial infarction and coronary death. ${ }^{3}$ A longitudinal Finnish study showed that the risk of metabolic syndrome at follow up was 2.5 times higher, relative to that observed at baseline, in women with depressive symptoms. ${ }^{10}$ A meta-analysis of longitudinal studies showed that depressed people were at significantly greater risk of developing obesity relative to nondepressed people. ${ }^{7}$ In addition, a large population-based study found that elderly people with depression exhibited significantly higher odds for receiving a diagnosis of hypertension, after adjustment for sociodemographic variables and comorbid chronic diseases, relative to those observed in people without depression. ${ }^{5}$ However, the results of the current study did not show a significant association between depression and diabetes. A longitudinal cohort study in the USA (follow-up 6.0 years) had reported that depressive symptoms predicted incident diabetes after adjusting for socio-economic factors, lifestyle factors, and metabolic covariates. ${ }^{6}$ In contrast, in another longitudinal study in the USA (mean follow-up 9.0 years), no association was observed between depressive symptoms and development of diabetes after adjusting for socio-demographic factors, known diabetes risk factors (body mass index and physical activity). ${ }^{19}$ The inconsistency between studies' results might be due to differences in the study methodology (e.g., longitudinal vs. crosssectional) and the methods adopted to measure depression (e.g., self-reported diagnosed depression vs. questionnaires for depressive symptoms) and diabetes (e.g., self-reported diagnosed diabetes vs. diabetes diagnosed by a physician, a blood sample test, or a prescription for diabetes medication).

The relationships between depression and alterations in autonomic nervous system and hypothalamic-pituitary-adrenal axis functioning, platelet activation, and inflammation could 
provide a biological explanation for the association between depression and CAD and/or CVRs. ${ }^{20}$ Another reason for this association could have been unhealthy lifestyle, including smoking, ${ }^{21}$ unhealthy dietary habits, ${ }^{22}$ and poor compliance with medical care, in people with depression. ${ }^{16}$

More importantly, the results study showed that women with both depression and CVRs, excluding diabetes, were significantly less likely to use healthcare services relative to those with CVRs alone. Mental illness is known to be a significant barrier to the receipt of medical services. For example, patients with mental illness were approximately twice as likely to report having been unable to obtain necessary medical care or experience a delay in seeking care because of cost, relative to those without mental illness, in a US sample. ${ }^{23}$ Druss et al. ${ }^{24}$ suggested several possibilities for lower likelihood of receiving medical treatment in individuals with mental disorders; one was low socioeconomic status, which could lead to a delay in seeking medical care because of cost, and another was that the cognitive or affective symptoms of mental disorders led to diminished capacity for medical decision making and complicated the provision of effective aftercare. ${ }^{24}$ In addition, individuals with depression could been less likely to use healthcare because of self-neglect, ${ }^{25}$ low self-efficacy, ${ }^{26}$ and a lack of autonomy in receiving healthcare. ${ }^{27}$ Moreover, doctors could have focus on depression management issues and neglected patients' physical health issues until they become more severe and obvious. ${ }^{28}$ For example, Moise et al. ${ }^{29}$ reported that clinical inertia was more likely in patients with hypertension who has also been diagnosed with depression, relative to those who had not been diagnosed with depression. In the current study, comorbid depression did not exert a significant effect on healthcare utilization in patients with severe and critical conditions such as $\mathrm{CAD}$ and diabetes; however, in patients with other CVRs, depression could have served as a barrier to healthcare service utilization. Consistent with our results, depressed patients reported poor access to primary care physicians in a Japanese study, ${ }^{30}$ and depression has been associated with lower cancer screening rates. ${ }^{28}$

The strengths of this study included the use of a large, nationally representative sample and examination of the effects of comorbid depression on healthcare service utilization in patients with CVRs or CAD, which was missing from the literature. Despite these strengths, the results should be interpreted in consideration of some limitations. First, the crosssectional design of the study did not allow us to infer a causal relationship between depression and CAD and/or CVRs. However, some previous studies have suggested a bidirectional relationship. ${ }^{9}$ Second, we used self-reported diagnosis and a single question, rather than a standardized assessment tool, to screen for depression; therefore, the data could have been subject to classification errors. ${ }^{31}$ However, self-reported diagnosis has generally shown good agreement with medical record data. ${ }^{32}$ Third, the questions regarding nonutilization of healthcare services were not specific to CAD or CVRs; therefore, whether the results reflected nonutilization of healthcare services for $\mathrm{CAD} / \mathrm{CVRs}$ or other medical problems remains unclear.

Considering the high rate of comorbid depression with $\mathrm{CAD}$ or CVRs, depression screening should be performed for patients with CVRs or $\mathrm{CAD}$ in primary healthcare institutions. In addition, considering the low levels of health service utilization in depressed patients, screening for common CVRs, such as obesity, hypertension, and dyslipidemia, should be provided for patients with depression in mental health care settings. To ensure that these interventions are successful, a comprehensive, collaborative care system should be established to deliver treatment required by people with depression and CAD or CVRs.

\section{Acknowledgments}

This study was supported by an Intramural Research Grant (No R2017A) from the National Center for Mental Health, Ministry of Health \& Welfare, Republic of Korea.

\section{REFERENCES}

1. World Health Organization. The Global Burden of Disease: 2004 Update. Geneva, Switzerland: World Health Organization; 2008.

2. Gallagher D, O’Regan C, Savva GM, Cronin H, Lawlor BA, Kenny RA. Depression, anxiety and cardiovascular disease: which symptoms are associated with increased risk in community dwelling older adults? J Affect Disord 2012;142:132-138.

3. Wu Q, Kling JM. Depression and the risk of myocardial infarction and coronary death: a meta-analysis of prospective cohort studies. Medicine 2016;95:e2815.

4. Wyman L, Crum RM, Celentano D. Depressed mood and cause-specific mortality: a 40-year general community assessment. Ann Epidemiol 2012;22:638-643.

5. Maatouk I, Herzog W, Böhlen F, Quinzler R, Löwe B, Saum KU, et al. Association of hypertension with depression and generalized anxiety symptoms in a large population-based sample of older adults. J Hypertens 2016;34:1711-1720.

6. Golden SH, Williams JE, Ford DE, Yeh HC, Paton Sanford C, Nieto FJ, et al. Depressive symptoms and the risk of type 2 diabetes: The Atherosclerosis Risk in Communities study. Diabetes Care 2004;27:429-435.

7. Blaine B. Does depression cause obesity?: a meta-analysis of longitudinal studies of depression and weight control. J Health Psychol 2008;13: 1190-1197.

8. van Reedt Dortland AK, Vreeburg SA, Giltay EJ, Licht CM, Vogelzangs $\mathrm{N}$, van Veen $\mathrm{T}$, et al. The impact of stress systems and lifestyle on dyslipidemia and obesity in anxiety and depression. Psychoneuroendocrinology 2013;38:209-218.

9. Pan A, Keum N, Okereke OI, Sun Q, Kivimaki M, Rubin RR, et al. Bidirectional association between depression and metabolic syndrome: a systematic review and meta-analysis of epidemiological studies. Diabetes Care 2012;35:1171-1180

10. Vanhala M, Jokelainen J, Keinanen-Kiukaanniemi S, Kumpusalo E, Koponen $\mathrm{H}$. Depressive symptoms predispose females to metabolic syndrome: a 7-year follow-up study. Acta Psychiatr Scand 2009;119:137-142. 
11. Sullivan M, Simon G, Spertus J, Russo J. Depression-related costs in heart failure care. Arch Intern Med 2002;162:1860-1866.

12. Egede LE. Major depression in individuals with chronic medical disorders: prevalence, correlates and association with health resource utilization, lost productivity and functional disability. Gen Hosp Psychiatry 2007; 29:409-416.

13. Baumeister H, Haschke A, Munzinger M, Hutter N, Tully PJ. Inpatient and outpatient costs in patients with coronary artery disease and mental disorders: a systematic review. Biopsychosoc Med 2015;9:11.

14. Jiang W, Alexander J, Christopher E, Kuchibhatla M, Gaulden LH, Cuffe MS, et al. Relationship of depression to increased risk of mortality and rehospitalization in patients with congestive heart failure. Arch Intern Med 2001;161:1849-1856.

15. Cherry SB, Benner JS, Hussein MA, Tang SS, Nichol MB. The clinical and economic burden of nonadherence with antihypertensive and lipid-lowering therapy in hypertensive patients. Value Health 2009;12:489497.

16. Gehi A, Haas D, Pipkin S, Whooley MA. Depression and medication adherence in outpatients with coronary heart disease: findings from the heart and soul study. Arch Intern Med 2005;165:2508-2513.

17. Park SJ, Roh S, Hwang J, Kim HA, Kim S, Lee TK, et al. Association between depression and metabolic syndorme in Korean women: results from the Korean national health and nutrition examination survey (2007-2013). J Affect Disord 2016;205:393-399.

18. Grundy SM, Cleeman JI, Daniels SR, Donato KA, Eckel RH, Franklin BA, et al. Diagnosis and management of the metabolic syndrome: an American Heart Association/National Heart, Lung, and Blood Institute scientific statement. Circulation 2005;112:2735-2752.

19. Saydah SH, Brancati FL, Golden SH, Fradkin J, Harris MI. Depressive symptoms and the risk of type 2 diabetes mellitus in a US sample. Diabetes Metab Res Rev 2003;19:202-208.

20. Dhar AK, Barton DA. Depression and the link with cardiovascular disease. Front Psychiatry 2016;7:1-9.
21. Glassman AH, Helzer JE, Covey LS, Cottler LB, Stetner F, Tipp JE, et al. Smoking, smoking cessation, and major depression. JAMA 1990;264:15461549.

22. Lang UE, Beglinger C, Schweinfurth N, Walter M, Borgwardt S. Nutritional aspects of depression. Cell Physiol Biochem 2015;37:1029-1043.

23. Druss BG, Rosenheck RA. Mental disorders and access to medical care in the United States. Am J Psychiatry 1998;155:1775-1777.

24. Druss BG, Bradford DW, Rosenheck RA, Radford MJ, Krumholz HM. Mental disorders and use of cardiovascular procedures after myocardial infarction. JAMA 2000;283:506-511.

25. Abrams RC, Lachs M, McAvay G, Keohane DJ, Bruce ML. Predictors of self-neglect in community-dwelling elders. Am J Psychiatry 2002;159:17241730 .

26. Mancuso CA, Rincon M, McCulloch CE, Charlson ME. Self-efficacy, depressive symptoms, and patients' expectations predict outcomes in asthma. Med Care 2001;39:1326-1338.

27. Mirowsky J, Ross CE. Control or defense? Depression and the sense of control over good and bad outcomes. J Health Soc Behav 1990;31:71-86.

28. Pirraglia PA, Sanyal P, Singer DE, Ferris TG. Depressive symptom burden as a barrier to screening for breast and cervical cancers. J Womens Health (Larchmt) 2004;13:731-738.

29. Moise N, Davidson KW, Chaplin W, Shea S, Kronish I. Depression and clinical inertia in patients with uncontrolled hypertension. JAMA Intern Med 2014;174:818-819.

30. Takahashi O, Deshpande GA, Ohde S. Despite high prevalence, depression is associated with less healthcare utilization N Japan (Abstract). J Gen Intern Med 2015;30:S136.

31. Yu M, Han K, Nam GE. The association between mental health problems and menstrual cycle irregularity among adolescent Korean girls. J Affect Disord 2017;210:43-48.

32. Kehoe R, Wu S, Leske MC, Chylack LT Jr. Comparing self-reported and physician-reported medical history. Am J Epidemiol 1994;139:813-818. 